\title{
The Relationship between Hwabyung Symptoms and Quality of Life among Middle-Aged Women
}

\author{
Kyoung-Mi Yang \\ Dept. of Nursing, Joongbu University, Korea \\ kmyang@joongbu.ac.kr
}

\begin{abstract}
The aim of this research is to examine the relationship between Hwabyung symptoms and life quality, and prepare basic data to improve life quality of middle-aged women. Research method: this research conducted a survey, and respondents of the survey were 230 middle-aged women conveniently sampled from two cities from March 10, to May 4, 2017. The survey data were analyzed using the SPSS 21.0 program. The average point of Hwabyung symptoms of respondents was 1.12 $561(0-4)$, and that of quality of life was $3.16 \pm 42$ (1-5). The variables significantly affecting Hwabyung symptoms were educational level $(F=7.14, p=.001)$, monthly income $(F=6.63, p<.001)$, and health condition $(F=12.10, p<.001)$. Quality of life was significantly and negatively correlated with Hwabyung symptoms $(r=-.204, p=.002)$. Data analysis showed that the lower education level is, the lower monthly income is, and the worse health condition is, the higher the symptom is, and that the higher educational level is, and the higher monthly income is, the higher life quality is. Therefore, to reduce Hwabyung symptoms, and improve life quality among middle-aged women, various educational means should be suggested and applied.
\end{abstract}

Keywords: Middle-aged woman, Hwabyung, Physical symptoms, Psychological symptoms, Quality of life

\section{Introduction}

Middle-aged women play various roles and perform various duties in their families and the society. Middle age is an important period in one's life cycle, covering as long as 30 years. From the perspective of family life cycle, while this period is characterized by reduction of burden of child rearing, it is also characterized by increase of burden of caring old parents and weighing of social responsibility. In particular, given that Korea is still full of Confucian patriarchal values, Korean middle-aged women are forced to sacrifice themselves for family members [1].

As a result, they suffer various types of psychological, physical stress, and such stresses are developed into what is called 'Hwabyung' in their minds [2]. Hwabyung is viewed in Korea as a disease or medical syndrome with various symptoms suffered mainly by middle-aged women who have to live under the patriarchal Korean culture [3][4][5]. Continuous inhibition of negative emotion is viewed as the main cause of the disease [5]. It is a psychological syndrome related to feeling unfair and angry with the incubation period of over 10 years [5]. Some people

Article history:

Received (August 5, 2019), Review Result (October 13, 2019), Accepted (November 28, 2019) 
go further, arguing that it is a psychiatric disorder with physical and behavioral symptoms of emotional condition related with rage [6][7].

Major symptoms of Hwabyung are as follows: physical symptoms like stuffiness, headache, body heat and facial heat, burning, palpitation, swelling heart, and lump in throat and neck; mental symptoms like gloom, anxiety, nervousness, and irritation [8]. Therefore, Hwabyung is either viewed as mental disorder similar to depressive disorder, panic disorder, and somatization disorder, etc., or as complex emotional disorder [9]. There have been qualitative and quantitative researches on Hwabyung since 1970.

Existing researches have found that major causes of Hwabyung are relations with husbands and families-in-law and conflicts with family members like brothers and sisters, followed by their social problems like financial difficulties, financial loss, or fraud [10][11]. But the problem is that those who suffer from symptoms of Hwabyung diagnose themselves as patients of Hwabyung, and are rarely likely to see a doctor [12]. Thus, it is necessary to help them to seek medical treatment. Hwabyung with its characteristic psychological and physical symptoms has negative effects on life quality.

Accordingly, this research intended to examine the effects of some general characteristics on symptoms of Hwabyung and life quality, and the relationship between symptoms of Hwabyung and life quality, and provide basic data to develop programs helping those suffering from Hwabyung.

\section{Methods}

\subsection{Research design}

This research is descriptive survey research to examine the effects of general characteristics on Hwabyung symptoms and life quality among middle-aged women, and the relationship between Hwabyung symptoms and life quality.

\subsection{Analytical objects and data collection}

The survey objects were 240 middle-aged women from 40 to 64 years old residing in D and $\mathrm{C}$ cities who understood the research goals and wanted to participate in the survey. Excluding 10 copies of the questionnaire which were not filled out completely, 230 copies were used for final analysis.

\subsection{Research tools}

\subsubsection{The scale to measure Hwabyung}

The scale to measure Hwabyung was developed by Kwon et al. [4]. The scale consists of 31 questions in total: 15 questions on the symptoms of the Hwabyung and 16 questions on Hwabyung personality. The questions on the symptoms of the Hwabyung were found by scholars to be useful to distinguish symptoms of depression and those of Hwabyung. Those questions are composed of 6 on physical symptoms and 9 questions on emotional symptoms. The scale consists of 5 points from 0 point (Not at all) to 4 points (Definitely yes). When they were developed, internal credibility of Hwabyung symptoms, Cronbach's alpha was .93, and Cronbach's alpha of this research was .92. 


\subsubsection{The scale to measure quality of life - Abbreviated form (who quality of Life-BREF)}

The scale of quality of life used by this research was originally made by WHO, and it is called WHOQOL-BREF [13]. The original WHOQOL-BREF was translated into Korean by Min et al. [14] and the Korean version WHOQOL-BREF passed the validity and reliability test. The Korean version WHOQOL-BREF consists of 26 questions, and, on each question, respondents are asked to choose from 1 point (Not at all) to 5 points (Definitely yes). The higher the value is, the higher quality of life is. In Min et al.'s research [9], reliability of the scale Cronbach's alpha was .90 , and that of this research was .88 .

\subsection{Data analysis}

The data were analyzed using the SPSS/PC 21.0 Statistical Program. First, frequency, percentage, mean, and standard deviation were acquired for variables on general characteristics and some other variables, and, to examine the effects of general characteristics on Hwabyung symptoms and quality of life, t-test and one-way ANOVA were done, and Scheffé test was done as post-hoc test. Correlations between Hwabyung symptoms and quality of life were analyzed with Pearson's correlation coefficient.

\section{Results}

\subsection{Hwabyung and quality of life}

The average score of Hwabyung symptoms of respondents was $1.12 \pm .61$ in the scale ranging from 0 to 4 . That of emotional symptoms, a sub-variable, was $1.06 \pm .66$, and that of physical symptoms, the other sub-variable, was $1.21 \pm .70$. The average score of quality of life was $3.16 \pm .42$ in the scale ranging from 1 to 5 [Table 1].

Table1. Descriptive data of Hwabyung and quality of life $(\mathrm{N}=230)$

\begin{tabular}{ccccc}
\hline \multirow{2}{*}{ Variable } & Classification & $\begin{array}{c}\text { Mean of Total value } \\
\mathrm{M} \pm \mathrm{SD}\end{array}$ & $\begin{array}{c}\text { Mean value } \\
\mathrm{M} \pm \mathrm{SD}\end{array}$ & Range \\
\hline \multirow{2}{*}{$\begin{array}{c}\text { Hwabyung } \\
\text { symptom }\end{array}$} & Psychological symptoms & $9.57 \pm 5.96$ & $1.06 \pm .66$ & \\
\cline { 2 - 5 } & Physical symptoms & $7.23 \pm 4.18$ & $1.21 \pm .70$ & $0-4$ \\
\hline Quality of life & Overall & $16.80 \pm 9.20$ & $1.12 \pm .61$ & $1-5$ \\
\hline
\end{tabular}

\subsection{Effects of general characteristics on Hwabyung and quality of life}

Survey respondents were 230 middle-aged women aged 40 to 64 . Their mean age was $52.06 \pm 6.65$. In the relationship between general characteristics and Hwabyung symptoms, educational level $(\mathrm{F}=7.14, \mathrm{p}=.001)$, monthly income $(\mathrm{F}=6.63, \mathrm{p}<.001)$, and health condition $(\mathrm{F}=12.10, \mathrm{p}<.001)$ were found to significantly affect Hwabyung symptoms. For example, middle school graduates tended to have more serious symptoms of Hwabyung than high school or college graduates. The lower monthly income was, the more severe symptoms were. For example, those whose monthly income was below 2 million won had more severe symptoms of Hwabyung than those whose income levels were higher. In health condition, those who answered "Not healthy," and "So so" tended to have more severe symptoms than those who answered "Healthy". The critical score to decide whether a person has Hwabyung is 30 points 
in total [4]. Among all the respondents of this research, 17 persons (7.4\%) were found to have Hwabyung, with their scores being 30 points or over.

Among the variables of general characteristics, it was found that educational level $(\mathrm{F}=15.87$, $\mathrm{p}<.001)$, and monthly income $(\mathrm{F}=3.38, \mathrm{p}=.010)$ had significant effects on quality of life. For example, quality of life of college graduates was higher than that of middle school and high school graduates. And, those whose monthly income was over 5 million won enjoyed higher quality of life than those whose monthly income was less than 2 million won [Table 2].

Table 2. Effects of general characteristics on Hwabyung symptoms and quality of life (N=230)

\begin{tabular}{|c|c|c|c|c|c|c|}
\hline \multirow{2}{*}{$\begin{array}{c}\text { General } \\
\text { characteristics }\end{array}$} & \multirow[b]{2}{*}{ Category } & \multirow{2}{*}{$\begin{array}{l}\mathrm{N}(\%) \text { or } \\
\mathrm{M} \pm \mathrm{SD}\end{array}$} & \multicolumn{2}{|c|}{ Hwabyung symptom } & \multicolumn{2}{|c|}{ Quality of life } \\
\hline & & & $\mathrm{M} \pm \mathrm{SD}$ & $\begin{array}{c}\text { t/F(p) } \\
\text { Scheffé }\end{array}$ & $\mathrm{M} \pm \mathrm{SD}$ & $\begin{array}{c}\text { t/F(p) } \\
\text { Scheffé }\end{array}$ \\
\hline Age(yr) & $\begin{array}{c}\text { Range } \\
\text { Mean age }\end{array}$ & $\begin{array}{c}40-64 \mathrm{yr} \\
52.06 \pm 6.65\end{array}$ & - & - & - & - \\
\hline \multirow{4}{*}{ Religion } & Catholic & $52(22.6)$ & $1.16 \pm .54$ & \multirow{4}{*}{$\begin{array}{c}.91 \\
(.150)\end{array}$} & $3.14 \pm .42$ & \multirow{4}{*}{$\begin{array}{l}1.25 \\
(.293)\end{array}$} \\
\hline & Christianity & $56(24.3)$ & $1.00 \pm .57$ & & $3.25 \pm .41$ & \\
\hline & Buddhism & $55(23.9)$ & $1.16 \pm .7 .0$ & & $3.12 \pm .46$ & \\
\hline & No religion & $67(29.1)$ & $1.16 \pm .63$ & & $3.12 \pm .39$ & \\
\hline \multirow{2}{*}{ Marriage } & Married & $187(81.3)$ & $1.09 \pm, 59$ & \multirow{2}{*}{$\begin{array}{l}-1.54 \\
(.344)\end{array}$} & $3.17 \pm .41$ & \multirow{2}{*}{$\begin{array}{c}1.07 \\
(.492)\end{array}$} \\
\hline & Single & $43(18.7)$ & $1.25 \pm .68$ & & $3.10 \pm .45$ & \\
\hline \multirow{3}{*}{$\begin{array}{c}\text { Educational } \\
\text { level }\end{array}$} & $\leq$ Middle school $^{\mathrm{a}}$ & $27(11.7)$ & $1.43 \pm .56$ & \multirow{3}{*}{$\begin{array}{c}7.14 \\
(.001) \\
a>c\end{array}$} & $3.04 \pm .26$ & \multirow{3}{*}{$\begin{array}{c}15.87 \\
(<.001) \\
a, b<c\end{array}$} \\
\hline & High School $^{\mathrm{b}}$ & $96(41.7)$ & $1.18 \pm .64$ & & $3.02 \pm .43$ & \\
\hline & $\geq$ College $^{c}$ & $107(46.5)$ & $0.98 \pm .56$ & & $3.32 \pm .40$ & \\
\hline \multirow{3}{*}{ Job } & Full time job ${ }^{a}$ & $109(47.4)$ & $1.14 \pm .63$ & \multirow{3}{*}{$\begin{array}{l}.58 \\
(.560)\end{array}$} & $3.20 \pm .40$ & \multirow{3}{*}{$\begin{array}{c}.82 \\
(.441)\end{array}$} \\
\hline & Part time $\mathrm{Job}^{\mathrm{b}}$ & $32(13.9)$ & $1.20 \pm .53$ & & $3.13 \pm .36$ & \\
\hline & Unemployed $^{c}$ & $89(38.7)$ & $1.07 \pm .62$ & & $3.12 \pm .47$ & \\
\hline \multirow{5}{*}{$\begin{array}{c}\text { Monthly income } \\
(10,000 \text { Won })\end{array}$} & $<200^{\mathrm{a}}$ & $39(17.0)$ & $1.41 \pm .65$ & \multirow{5}{*}{$\begin{array}{c}6.63 \\
(<.001) \\
a>d, e \\
c>e\end{array}$} & $3.02 \pm .33$ & \multirow{5}{*}{$\begin{array}{c}3.38 \\
(.010) \\
a<e\end{array}$} \\
\hline & $200 \leq \sim<300^{b}$ & $48(20.9)$ & $1.16 \pm .69$ & & $3.09 \pm .50$ & \\
\hline & $300 \leq \sim<400^{c}$ & $51(22.2)$ & $1.27 \pm .52$ & & $3.15 \pm .43$ & \\
\hline & $400 \leq \sim<500^{d}$ & $30(13.0)$ & $0.93 \pm .44$ & & $3.17 \pm .37$ & \\
\hline & $\geq 500^{\mathrm{e}}$ & $62(27.0)$ & $0.88 \pm .56$ & & $3.30 \pm .39$ & \\
\hline \multirow{3}{*}{$\begin{array}{l}\text { Subjective health } \\
\text { condition }\end{array}$} & Healthy $^{a}$ & $94(40.9)$ & $0.91 \pm .53$ & \multirow{3}{*}{$\begin{array}{c}12.10 \\
(<.001) \\
a<b, c\end{array}$} & $3.21 \pm .51$ & \multirow{3}{*}{$\begin{array}{c}1.31 \\
(.273)\end{array}$} \\
\hline & So so $^{\mathrm{b}}$ & $111(48.3)$ & $1.23 \pm .61$ & & $3.13 \pm .33$ & \\
\hline & Not healthy ${ }^{\mathrm{c}}$ & $25(10.9)$ & $1.45 \pm .61$ & & $3.10 \pm .43$ & \\
\hline \multirow{2}{*}{$\begin{array}{l}\text { Hwabyung } \\
\text { symptoms }\end{array}$} & $<30$ & $213(92.6)$ & - & - & & \\
\hline & $>30$ & $17(7.9)$ & - & - & & \\
\hline
\end{tabular}

\subsection{Relationship between Hwabyung symptoms and quality of life}

It was found that quality of life is negatively related with Hwabyung symptoms [Table 3]. 
Table 3. Correlation between Hwabyung and quality of life $(\mathrm{N}=230)$

\begin{tabular}{|c|c|c|}
\hline Variable & Hwabyungsymptom & Quality of life \\
\hline Hwabyung symptom & 1 & - \\
\hline Quality of Life & $-.204(\mathrm{p}=.002)$ & 1 \\
\hline
\end{tabular}

\section{Discussion}

This research identified the effects of general characteristics on Hwabyung symptoms and quality of life among middle-aged women in Korea. The research was performed to know what nursing intervention might be effective to improve their quality of life of those middle-aged women suffering from symptoms of Hwabyung. The findings were as follows. The mean value of Hwabyung symptoms of respondents was 1.12 in the scale ranging from 0 to 4 (the mean cumulative value was 16.80 in the scale whose top point is 60 ). The value was lower than the corresponding mean value 1.27 in the research of Kim \& Lee [15]. The cumulative value of 16.80 was lower than that of 18.38 of Kwon et al [4], but higher than 13.11, the corresponding value in Park and Choi [16]. The mean values of symptoms of the two sub-variables of Hwabyung symptoms, psychological symptoms and physical symptoms were 1.06 and 1.21 , which were lower than corresponding values in Kim \&Lee [15]. This research and previous researches have shown that the value of physical symptoms is higher than that of psychological symptoms [15], proving that Hwabyung tends to express by physical symptoms rather than psychological symptoms. When we set the critical value of Hwabyung as 30 points [4], the proportion of respondents in this research belonging to this category was $7.4 \%$, which was lower than $8.1 \%$ in Kim \& Lee [15], but higher than $4.2 \%$ in Min [17].

The mean value of quality of life in this research was 3.16 in the scale ranging from 0 to 5 , which was lower than 3.28 in Kim [18], and 3.2 in Chung [19], but higher than 3.10 in Yang [20]. We can conclude that quality of life the respondents of this research enjoy is not different from the quality of life other groups of respondents in other studies did.

Among the variables related with general characteristics, educational level, monthly income, and health condition were significantly related with Hwabyung symptoms. That is, the lower educational level was, the smaller monthly income was, and the worse health condition was, the higher Hwabyung symptoms were. Such findings are consistent with the findings of Min [5] that Hwabyung tended to occur among middle-aged women, particularly those lower in socio-economic statuses. We can expect that those women with lower educational level have limited opportunities to choose jobs, impairing their economic stability, which deprives them of the time to take care of their own health. Imprisoned in such a vicious cycle, they lose the ability to regulate or express their emotions, or suppress such emotions, succumbing to Hwabyung symptoms.

Among variables related with general characteristics, educational level and monthly income were significantly related with quality of life. That is, the higher educational level was, and the higher monthly income was, the higher quality of life was. It was found that low educational level and bad health condition not only aggravate Hwabyung symptoms, but negatively affect quality of life. Such findings prove that common causes of Hwabyung symptoms and quality of life are educational level and monthly income, which means that low educational level and low monthly income increase Hwabyung symptoms and decrease quality of life.

Consequently, to improve quality of life, we should search for a concrete way to reduce Hwabyung symptoms. That is, we need a way to provide middle-aged women with educational 
opportunities. Various educational opportunities will provide them with job opportunities, which will increase economic stability, which will improve their quality of life.

Previous researches have suggested various ways to relieve Hwabyung symptoms and improve life quality such as emotional support of family members and friends and psychological counseling. But, the most essential treatment of Hwabyung may be the method to efficiently respond to and manage stresses [9].

Consequently, to help patients with Hwabyung to respond to stresses can be the way to prevent cumulated stresses from being developed into Hwabyung or other physical and mental disorders.

Finally, if those with Hwabyung symptoms have difficulty in controlling the symptoms, they should be given psychological counseling or medical help. In particular, systematic psychological counseling where family members of those with Hwabyung symptoms are included can be very effective in eliminating causes of the symptoms and improving life quality of middle-aged women.

\section{Conclusion and suggestions}

This research was performed to examine Hwabyung symptoms and quality of life among middle-aged women, and identify effects of general characteristics on Hwabyung symptoms and quality of life, and to provide basic data for developing educational programs to improve quality of life of those women. It was found that low educational level, low monthly income, and bad health condition aggravate Hwabyung symptoms, and that high educational level and monthly income increase quality of life. The common variables affecting Hwabyung symptoms and quality of life are educational level and monthly income.

As this research is based on the survey to middle-aged women in only two cities, the findings of this research have limits to be generalized. In the future researches on how to improve quality of life of those women, the survey should be done with the national basis, and more variables should be applied related with Hwabyung.

Based on the findings of this research, this research wants to offer the followings. First, it is necessary to do researches to identify various causes of Hwabyung. Second, it is also necessary to do qualitative survey through in-depth interview. Third, by identifying causes of Hwabyung through cross-sectional analysis, we can develop educational programs to reduce stresses.

\section{References}

[1] M. J. Kim and M. H. Hyun, "The relationship between stress, social support, self-esteem and Hwabyung of men,” The Korean Journal of Health Psychology, vol.15, no.1, pp.19-33, (2010)

[2] J. S. Park and Y. S. Lyu, "A bibliographic study about the meaning of Hwa and Hwabyung," J of Oriental Neuropsychiatry, vol.8, no.1, pp.183-200, (1997)

[3] H. I. Kwon., S. Y. Lee, and J. H Kwon, "Hwabyung symptoms and anger expression in relation to submissive behavior and external entrapment in married couple," The Korean journal of Woman Psychology, vol.17, no.4, pp.579-595, (2012)

[4] J. H. Kwon., J. W. Kim., D. G. Park., M. S. Lee., S. K Min, and H. I. Kwon, "Developmentand validation of the Hwabyung scale," The Korean Journal of Clinical Psychology, vol.27, no.1, pp.237-252, (2008)

[5] S. K. Min, "A study of the concept of Hwabyung," J Korean Neuropsychiatr Assoc, vol.28, no.4, pp.604-616, (1989)

[6] S. K. Min, "Hwabyung and Art therapy," Journal of the Korean academy of clinical art therapy, vol.4, no.2, pp.61-67, (2009) 
[7] S. K. Min., S. Y. Suh., Y. K. Cho., J. E. Huh, and K. J. Song, "Development of Hwabyung scale and research criteria of Hwa-Byung," J Korean Neuropsychiatr Assoc, vol.48, pp.77-85, (2009)

[8] J. W. Kim, J. H. Lee, S. G. Lee, H. J. Eom, and W. W. Whang, "A clinical study on Hwabyung with HwaByung model of oriental medicine," The Korean journal of stress research, vol.4, no.2, pp.23-32, (1996).

[9] H. J. Cho, J. W. Kim, and S. Y. Son, "A qualitative study about the therapeutic process of loving-kindness meditation for patients with Hwabyung," The Korean Journal of Counselling and Psychotherapy, vol.25, no.3, pp.425-448, (2013)

[10] J. W. Kim, "Hwa-Byung-assessment and Management," The Korean journal of stress research, vol.13, no.2, pp.43-47, (2005)

[11] S. J. Park and E. Y. Choi, "Content analysis of Hwabyung awareness in middle-aged women," Journal of the Korea Academia-Industrial cooperation Society, vol.16, no.2, pp.1304-1317, (2015)

[12] Y. Choi, K. Y. Pang, and S. Kim, “A transcultural model of Hwabyung: A grounded theory,” J of Korean Academy of Psychiatric and Mental Health Nursing, vol.15, no.4, pp.508-512, (2006)

[13] "The WHOQOL group: Development of the world health organization WHOQOL-BREF quality of life assessment," Psycho Med, vol.28, pp.551-558, (1998)

[14] S. K. Min., C. I. Lee., K. I. Kim., S. Y. Suh, and D. K. Kim., "Development of Korean version of WHO quality of life scale abbreviated version (WHOQOL-BREF), J Journal of Korean Neuropsychiatric Association, vol.39, no.3, pp.571-579, (2000)

[15] N. S. Kim and K. E. Lee., "Gender differences in factors affecting Hwabyung symptom with middle-age people," Journal of Korean Acad Fundam Nursing, vol.19, no.1, pp.98-105, (2012)

[16] S. J. Park and E. Y. Choi, "Relationships among Hwabyung, depression and quality of life in middle aged women," The Journal of Asian Woman, vol.53, no.2, pp.167-188, (2014)

[17] S. K. Min, K. Namkoong, and H. Y. Lee., “An epidemiological study on Hwabyung,” J Korean Neuropsychiatr Asso, vol.29, no.4, pp.867-874, (1990)

[18] K. H. Kim, "The factors influencing to quality of life of middle-aged women," Journal of the Korean Data Analysis Society, vol.18, no.1B, pp.497-508, (2016)

[19] M. S. Chung, "Resilience, coping methods and quality of life in middle-aged women," J of Korean Academic of Psychiatric and Mental Health Nursing, vol.20, no.4, pp.345-354, (2011)

[20] K. M. Yang, "The effect of depression, life stress and resilience on quality of life in middle aged women," J korean Acad Soc Home Care Nurs, vol.22, no.2, pp.300-309, (2015) 
The Relationship between Hwabyung Symptoms and Quality of Life among Middle-Aged Women

This page is empty by intention. 